\title{
QUEEN'S
UNIVERSITY
BELFAST
}

\section{Investigation of point cloud registration uncertainty for gap measurement of aircraft wing assembly}

Yang, Y., Jin, Y., Price, M., Abdelal, G., Higgins, C., \& Maropoulos, P. (2021). Investigation of point cloud registration uncertainty for gap measurement of aircraft wing assembly. Paper presented at 2021 IEEE International Workshop on Metrology for AeroSpace, Naples, Italy. https://www.metroaerospace.org/home

\section{Document Version:}

Peer reviewed version

Queen's University Belfast - Research Portal:

Link to publication record in Queen's University Belfast Research Portal

Publisher rights

Copyright 2021 the Author.

\section{General rights}

Copyright for the publications made accessible via the Queen's University Belfast Research Portal is retained by the author(s) and / or other copyright owners and it is a condition of accessing these publications that users recognise and abide by the legal requirements associated with these rights.

Take down policy

The Research Portal is Queen's institutional repository that provides access to Queen's research output. Every effort has been made to ensure that content in the Research Portal does not infringe any person's rights, or applicable UK laws. If you discover content in the Research Portal that you believe breaches copyright or violates any law, please contact openaccess@qub.ac.uk. 


\section{Investigation of point cloud registration uncertainty for gap measurement of aircraft wing assembly}

\author{
Ye Yang \\ School of mechanical and aerospace \\ engineering \\ Queen's University Belfast \\ Belfast, UK \\ yyang36@qub.ac.uk \\ Gasser Abdelal \\ School of mechanical and aerospace \\ engineering \\ Queen's University Belfast \\ Belfast, UK \\ g.abdelal@qub.ac.uk
}

\author{
Yan Jin \\ School of mechanical and aerospace \\ engineering \\ Queen's University Belfast \\ Belfast, UK \\ y.jin@qub.ac.uk \\ Colm Higgins \\ Northern Ireland Technology Centre \\ Queen's University Belfast \\ Belfast, UK \\ c.j.higgins@qub.ac.uk
}

\author{
Mark Price \\ School of mechanical and aerospace \\ engineering \\ Queen's University Belfast \\ Belfast, UK \\ M.Price@qub.ac.uk \\ Paul Maropoulos \\ School of mechanical and aerospace \\ engineering \\ Queen's University Belfast \\ Belfast, UK \\ Paul.Maropoulos@qub.ac.uk
}

\begin{abstract}
Point cloud registration is necessary to join multiple laser scanned data, but researchers have not treated point cloud registration in aircraft assembly in much detail. When applying laser scan metrology into industries, the effects of registration uncertainty on high precision assembly accuracy are not negligible. Based on the use of laser scanner and commercial software PolyWorks, this study investigates the registration uncertainty between part-level and assembly-level point cloud data in aircraft wing assembly. A spar-and-skin assembly with sphere artefacts is used as a case study. Registration uncertainty in gap measurement is also investigated. Results show that: (1) the use of sphere artefacts cannot improve registration accuracy in PolyWorks, but can improve efficiency. (2) Registration process could bring errors and these errors would be affected by the parameters settings during data processing. (3) Systematic errors would be associated with both part-level and assembly-level measurements, and calibration should be applied to eliminate their effects on the measurement of the desired dimensions, i.e. the gap size in this case. It is concluded that laser scanner and computational software can be used for high precision assembly, and evaluating registration uncertainty is a crucial step to improve assembly accuracy.
\end{abstract}

Keywords-point cloud registration, laser scanner, gap measurement, sphere target, aircraft wing assembly

\section{INTRODUCTION}

Gap measurement in aircraft assembly is necessary to maintain final product quality. However, existing manual gap measurement process currently used in aerospace industry is rather time-consuming and labour-intensive. Laser scanner is a powerful tool to capture detailed point cloud information of an object, which could potentially be used to automate the measurement process. It has been applied widely in the field of civil engineering [1] and remote sensing. There is also a trend to apply a metrology instrument into industrial product lines, e.g. aircraft, automobile and ship, and virtual factory [2].

\section{A. Key challenges when applying metrology instrument into industries}

When applying the instrument in the field of mechanical engineering, the uncertainty issue becomes very critical. Minnetti et al. [3] proposed a laser triangulation measurement device to assess gap and flush in car assembly. Uncertainties of both this laser triangulation measurement device and an operator were assessed by Baleani et al. [4] using Type A

This paper is funded by the China Scholarship Council, Queen's

University Belfast, and the Engineering and Physical Sciences Research

Council (EPSRC) UK. analysis and a Gauge R\&R crossed study. Results show that the gap's expanded uncertainty is $\pm 0.17 \mathrm{~mm}$ while for a flush is $\pm 0.13 \mathrm{~mm}$. Compared with manual measurement uncertainty, which is $\pm 0.5 \mathrm{~mm}$, the use of this laser scanner can improve gap and flush measurement accuracy. This indicates that the measurement process uncertainty is worth being evaluated, especially for laser-based instruments.

Another critical issue is to register different point clouds. Due to the laser-based metrology instrument's limited scanning volume, multiple scans are required to cover the whole object. Each scan is obtained at the local geometrical reference frame. Therefore to obtain a full view of the objects, multiple scans should be joined together, and this process is called registration. Registration can be carried out between different data sets from the same scanner or between different data sets from different scanners [5].

Registration has been used in the research of aircraft wreckage reconstruction and the general fastener assembly process. In an investigation of point cloud registration in the aircraft industry, Sun et al. [6,7] developed a system to splice an aircraft's wreckage using 3D point clouds and measurement technology. Results show that their coarse registration accuracy is $0.6 \mathrm{~mm}$, and the acceptable registration accuracy is $0.2 \mathrm{~mm}$. Wang et al. [8] proposed a general density-invariant framework for aircraft point cloud registration. Results show their method has better accuracy $(0.6 \mathrm{~mm}-1.0 \mathrm{~mm})$ evaluated by Root Mean Square Error (RMSE) than other studies [9-11]. Though the accuracy was improved, the proposed method was used for whole scanning aircraft rather than specific parts. Xu et al. [12] proposed a registration methodology for fastener assembly, in which local geometric feature and Iterative closest point (ICP) algorithm are used. The registration method was used between the scanned data and the CAD model. Results show that the proposed method has better efficiency compared with using the ICP algorithm only. However, the uncertainty of the proposed registration method was not disclosed.

\section{B. Current research trends in point cloud registration}

A considerable amount of literature has been published on point cloud registration. This literature has highlighted several topics, including the proposal of different registration algorithms [13-15] and local feature descriptors [16], improvement of registration efficiency [1], error analysis [17- 
22], development of new targets [23, 24], and automatic registration [25-27].

Registration can be categorised into coarse registration and fine registration. Feature-based coarse registration methods include point-based, line-based, and surface-based coarse registration method [13]. Fine registration methods include ICP [15] and its variants, random sample consensus methods (RANSAC), normal distributions transform (NDT) methods and methods with auxiliary data [13].

To date, several studies have investigated the performance of registration algorithms [19], and most researchers attempted to evaluate it using different parameters, including maximum/minimum/mean offset distance, standard deviation, Root Mean Square (RMS)/Root Mean Square Error (RMSE) $[17,18]$ and efficiency of registration. Ragendra et al. [18] used RMS for accuracy evaluation of CloudCompare and ICP variants, including Brute Force, KDTree, Partial Overlap in MATLAB. Results show that the most accurate method is the Brute Force, followed by KDTree and Partial Overlap. CloudCompare is ranked the last one. Bueno et al. [20] used the Monte Carlo method to assess the input point clouds' variations for the fine registration process. Results show an increasing trend with the coarse registration error for both translation and rotation.

Some researchers also defined new metrics for performance analysis of registration methods. Li et al. [22] conducted point-to-point and point-to-plane ICP algorithm practical range analysis using RMS and the relative RMS. Attia et al. [21] evaluated the performance of point-to-point ICP, point-to-plane ICP, NDT, Softassign, and RANSAC. Five new metrics were defined in this research, including Speed Accuracy product, Rotation Impact ratio, Translation Impact ratio, Noise Impact Ratio, and Sampling Impact ratio. Results show that: (1) the use of the sampling clouds algorithm leads to a worse accuracy. (2) ICP requires to use of all the point cloud points. (3) ICP is generally the best algorithm, whereas NDT and Softassign usually are in the last rank. (4) ICP point-to-point is more stable than ICP point-toplane, while the latter is more accurate than the former in terms of resistance on large rotations or translations.

Several studies have revealed that using targets can help automate the registration process of large point clouds. One study by Becerik-Gerber et al. [24] examined three types of targets and their effects on registration accuracy and efficiency of two building scans, including paper target, paddle target and sphere target. Results show that the sphere targets had the best performance in all tests. However, their research is not relevant to the aircraft assembly process in which accuracy is paid extreme attention. Other studies [2527] have also focused on using sphere targets to automate registration.

\section{Knowledge gaps and problem statement}

During the survey of the literature, the authors found the following knowledge gaps:

- Point cloud registration is a necessary step when applying laser scanners to industries. Current studies on registration are mainly in the fields of civil engineering, computer vision and aircraft reconstruction. Point cloud registration has not been well investigated in high precision assembly, e.g. aircraft assembly.
- Existing registration research are using two pointclouds of one object. Given that the assembly process consists of individual parts and assemblies, registration between part-level and assembly-level point clouds should be investigated.

- Registration will bring effects on the accuracy of the cloud data matching for assemblies. Besides, registration targets can be used for accurately aligning different point clouds. Given that aircraft wing assembly has large wing skin and spars, it must be subdivided into small parts. Therefore, targets should be used in order to identify different parts in the virtual environment. The influence of its use on registration uncertainty in aircraft assembly currently remains unclear.

For high precision assembly, the effects of registration on assembly accuracy may not be negligible. Therefore, to address this problem, this study investigates the registration uncertainty between part-level and assembly-level point clouds data in the aircraft wing assembly using commercial software package PolyWorks. Taking gap measurement as case studies, the effects of the use of sphere target in registration and gap analysis are also studied.

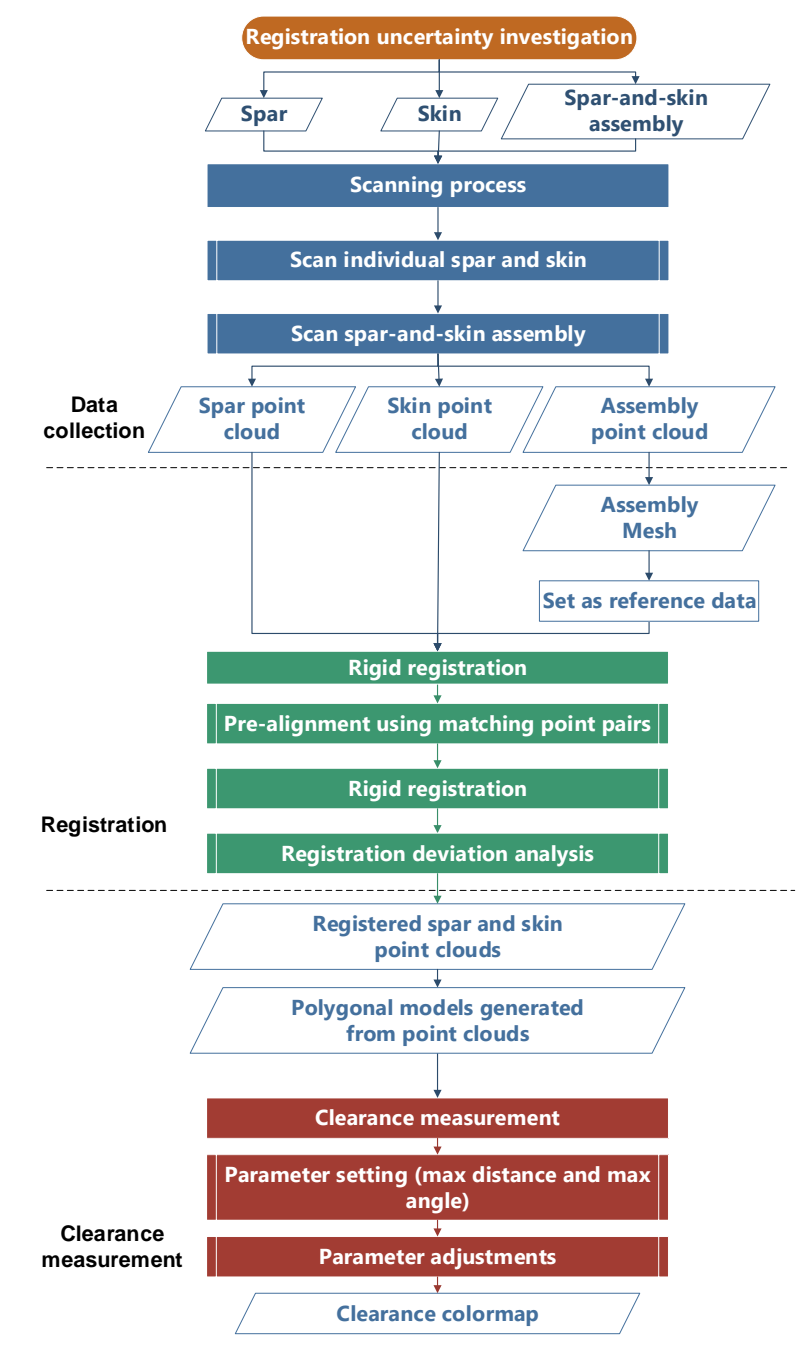

Fig. 1. Flowchart of the method 

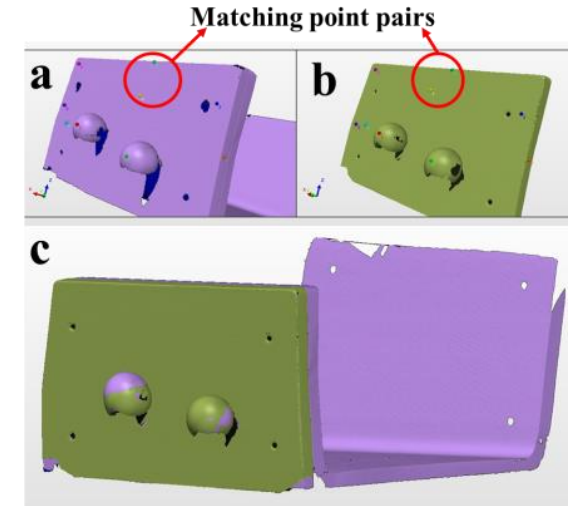

Fig. 2. Pre-alignment and registration (a) assembly point cloud with matching points; (b) skin point cloud to be aligned with matching points; (c) registration results.

\section{REGISTRATION IN POLYWORKS}

\section{A. Registration procedure in PolyWorks}

The registration procedure implemented in PolyWorks is shown in Fig.1.

In data collection, the laser scanner scans and collects spar, skin, and assembly point clouds. The assembly point cloud is then converted to polygonal mesh and set as reference data. Individual spar and skin point clouds are then registered to the reference data, i.e. the assembly polygonal mesh. Firstly, prealignment is carried out, in which the user picks matching point pairs between two datasets, in order to let the software calculate a coarse rigid transformation matrix, as shown in Fig. 2. Based on pre-alignment, the fine registration is conducted. In the case studies, the parts are assumed as rigid parts in order to simplify the problems, so that rigid registration can be carried out.

In this study, registration error are evaluated by deviations between points in registered point clouds and assembly polygonal mesh surfaces (reference data). Standard deviation is used to reflect registration error, and registration efficiency is indicated by the iteration times of registration.

This study takes gap measurement as a practical application of registration, whose procedure is also shown in Fig. 1. Firstly, both registered spar and skin point clouds are converted to polygonal meshes. Then the gap between spar and skin mating surfaces is measured in PolyWorks. The user should adjust two parameter settings in order to get better results, i.e. max distance and max angle. Finally, a colormap of the gap is obtained.

In the overall workflow, laser scanner systematic error can affect data analysis results. Besides, using sphere artefacts as registration targets may also influence registration results. Therefore, the systematic error analysis of the laser scanner is carried out (Section III A), and the impacts of using sphere targets on registration (Section III B) and gap measurement (Section III C) are also investigated.

\section{EXPERIMNETS AND ANALYSIS}

\section{A. Systematic error evaluation for laser scanner}

\section{1) Experiment workflow}
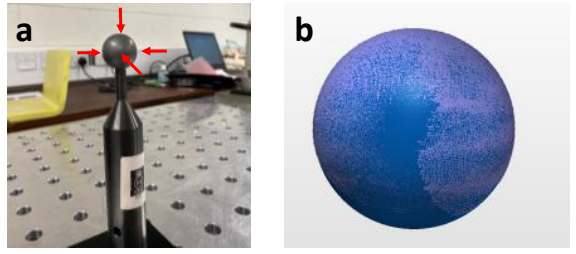

Fig. 3. Laser scanner systematic error evaluation test: (a) calibrated sphere and scanning directions; (b) sphere point cloud data (purple) and the extracted sphere feature (blue).

TABLE I. STSTTEMATIC ERROR EVALUATION TEST RESULTS (UNIT:MM)

\begin{tabular}{|c|c|c|c|c|}
\hline & $\begin{array}{c}\text { Calibrated } \\
\text { Diameter }\end{array}$ & $\begin{array}{c}\text { Measured } \\
\text { Diameter }\end{array}$ & Error & $\begin{array}{c}\text { Error } \\
\text { percentage }\end{array}$ \\
\hline Mean & 25.400 & 25.3261 & -0.0739 & $0.291 \%$ \\
\hline
\end{tabular}

A laser scanner (Romer Absolute Arm 7325SEI) with an accuracy of $\pm 0.084 \mathrm{~mm}$ stated by the manufacturer is used to collect data in this study. In this test, a calibrated sphere artefact (Fig. 3a) with a diameter of $25.400 \mathrm{~mm}$ was placed randomly at 55 different locations within the measuring volume of Romer Arm. The laser scanner scanned the sphere from five directions (one from top-view and four from sideviews, see Fig. 3a). The scanned sphere point clouds were processed in PolyWorks to extract sphere features using leastsquare algorithm (Fig. 3b). Finally, measured diameters were compared with the calibrated diameter to evaluate laser scanner systematic error. Table I shows the error analysis between measured and calibrated diameters. The mean error is $-0.0739 \mathrm{~mm}$, which means the laser scanner has a systematic error (denoted as $E_{s}$ ) of $-0.0739 \mathrm{~mm}$, and its accuracy is within the range stated by the manufacturer $( \pm 0.084 \mathrm{~mm})$.

\section{2) Systematic error elimination}

The systematic error occurs in the point clouds of the two individual parts and the assembly. If the scanned data are used for downstream gap measurement, these systematic errors will propagate to the geometry to be measured, i.e. the gap. It is assumed here that the systematic error of laser scanner is always the same for scanning parts or assemblies in any shapes and sizes.

As shown in Fig. 4, the measured data of spar, skin, and the assembly are denoted as $D_{\text {spar,meas }}, D_{\text {skin,meas }}$, and $D_{\text {assem,meas }}$, respectively. The measured gap is denoted as $D_{c, \text { meas }}$. The calibrated results of measurement data are denoted as $D_{\text {spar }}, D_{\text {skin }}, D_{\text {assem }}$, and $D_{c}$, respectively. The measurement results contain systematic errors, which means:

$$
D_{\text {spar,meas }}=D_{\text {spar }}+E_{S}
$$

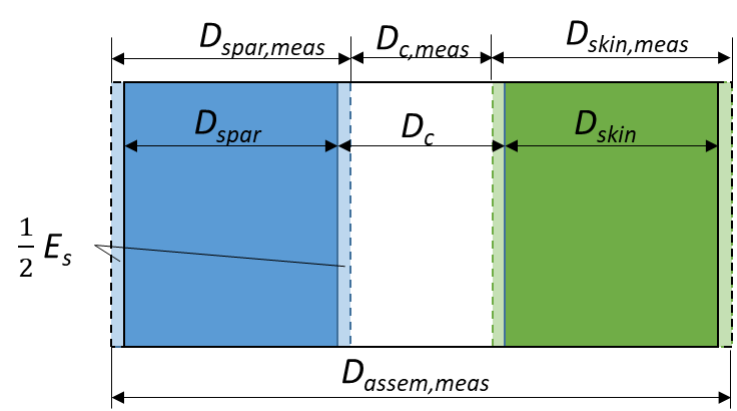

Fig. 4. Illustration of laser scanner systematic error 


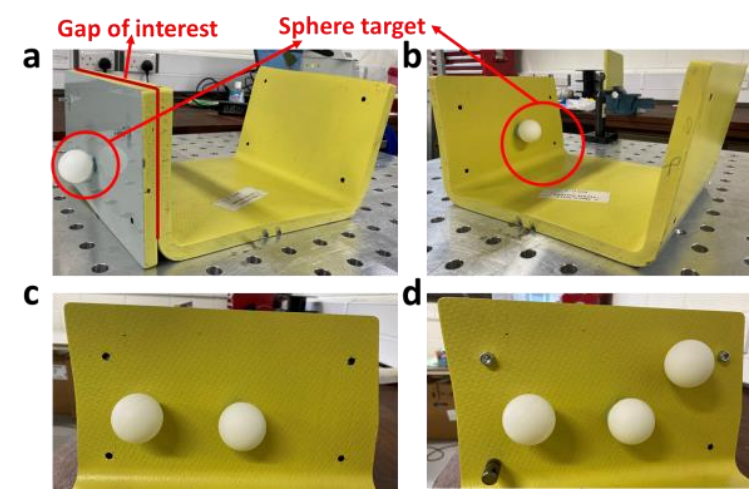

Fig. 5. Spar-and-skin assembly: (a) gap of interest; (b) with one sphere target; (c) with two sphere targets; (d) with three sphere targets.

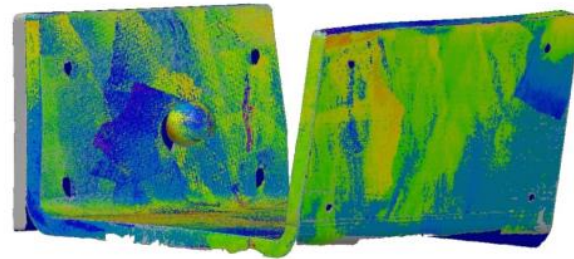

Fig. 6. Registration deviation measurement

TABLE II. MEAN STANDARD DEVIATION AND ITEATIONS OF REGISTRATION TESTS

\begin{tabular}{|c|c|c|}
\hline Target number & $\begin{array}{c}\text { Mean standard } \\
\text { deviation }(\mathbf{m m})\end{array}$ & Mean Iterations \\
\hline 0 & 0.0382 & 10.3333 \\
\hline 1 & 0.0393 & 9.3333 \\
\hline 2 & 0.1818 & 6.6667 \\
\hline 3 & 0.1790 & 6 \\
\hline
\end{tabular}

$$
\begin{aligned}
D_{\text {skin,meas }} & =D_{\text {skin }}+E_{s} \\
D_{\text {assem,meas }} & =D_{\text {assem }}+E_{s}
\end{aligned}
$$

Since $D_{c}$ and $D_{c, \text { meas }}$ can be described as follows:

$$
\begin{gathered}
D_{c}=D_{\text {assem }}-D_{\text {spar }}-D_{\text {skin }} \\
D_{c, \text { meas }}=D_{\text {assem,meas }}-D_{\text {spar,meas }}-D_{\text {skin,meas }}
\end{gathered}
$$

Substitute equation (1) - (4) to equation (5),

$$
D_{c}=D_{c, \text { meas }}+E_{S}
$$

Therefore, the final gap measurement result should add the systematic error in order to obtain calibrated results and eliminate systematic error of the scanner.

\section{B. Registration uncertainty analysis with sphere targets}

In this case study, the impacts of sphere targets on registration uncertainty are investigated.

A dummy composite aircraft wing spar-and-skin assembly (Fig. 5) was used. 1, 2, or 3 spheres were attached to the spar and skin non-mating surfaces (see Fig. 5), which were used for registration steps.

As shown in Fig. 1, the skin and spar were scanned individually. Then the spar and skin were assembled using fasteners to mimic aircraft wing spar-and-skin assembly, and scanned by the laser scanner. To increase the reliability of measures, each group of data were scanned three times in the case studies. The noise point cloud data outside the envelope of the scanned parts were removed. The registration procedure was carried out as stated in Section II. In pre-alignment process, point pairs were picked on both component surfaces and sphere targets. After registration, the deviations between spar point cloud and assembly mesh, and between skin point cloud and assembly mesh were calculated (Fig. 6).

Table II shows the results of the registration analysis. It can be seen that the standard deviation of registration increases with the use of sphere targets. However, registration iteration times decreases. This means that though the use of sphere targets cannot help improve registration accuracy, it can help improve the registration efficiency by reducing iterations in PolyWorks. This indicates that registration targets can be used in a future factory to help operators identify different mating surfaces of different component scanned data.

\section{Gap measurement tests}

After registration, the spar and skin point clouds were converted into polygonal meshes to measure the gaps between them, as shown in Fig. 1.

The first part of this case study investigates the impacts of two settings (max angle and max distance) on gap measurement results. Secondly, the impacts of the use of sphere targets on gap measurement results are investigated. Finally, PolyWorks measurement results are calibrated by laser scanner systematic error obtained in the previous test, and compared with manual measurement results by feeler gauges.

1) Impacts of two settings: max distance and max angle

In PolyWorks, the user has to set max distance and max angle when measuring gaps. Fig. 7 shows the brief theory for gap measurement. $P$ (red dot) is one of the triangle vertices on the spar polygon mesh. $Q$ is a matching point of $P$ on the skin mating surface of skin polygon mesh along the vertex normal

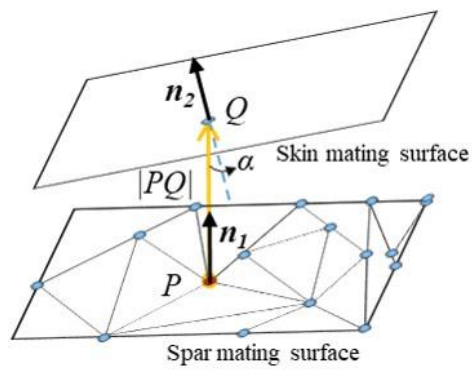

Fig. 7. Distance measurement of polygonal meshes
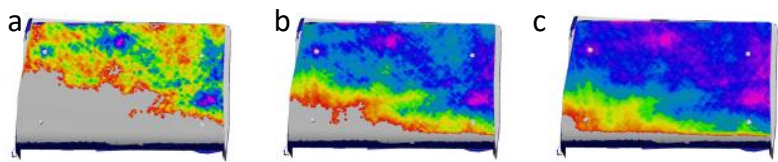

Fig. 8. Max distance: (a) $0.5 \mathrm{~mm}$; (b) $1 \mathrm{~mm}$; (c) $1.5 \mathrm{~mm}$
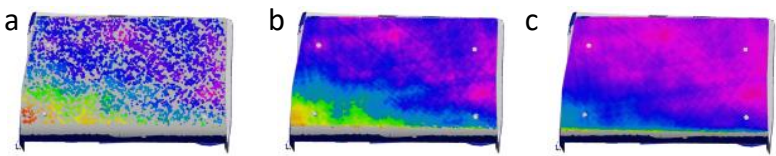

Fig. 9. Max angle: (a) 1 degree; (b) 5 degree; (c) 15 degree 
TABLE III. GAP MEASUREMENT RESULTS COMPARASON (UNIT:MM)

\begin{tabular}{|c|c|c|c|}
\hline $\begin{array}{c}\text { Target } \\
\text { number }\end{array}$ & $\begin{array}{c}\text { Mean standard } \\
\text { deviation }\end{array}$ & $\begin{array}{c}\text { Mean } \\
\text { Error }\end{array}$ & $\begin{array}{c}\text { Mean Error after } \\
\text { calibration }\end{array}$ \\
\hline 0 & 0.2538 & -0.0822 & 0.0082 \\
\hline 1 & 0.2535 & 0.1065 & 0.0326 \\
\hline 2 & 0.2545 & 0.1125 & 0.0386 \\
\hline 3 & 0.2470 & 0.0465 & -0.0274 \\
\hline
\end{tabular}

vector $\boldsymbol{n}_{\boldsymbol{1}}$. The gap thickness at $P$ is defined as the distance between $P$ and $Q,|P Q|$. Let $\boldsymbol{n}_{2}$ represent the surface normal vector at $Q$. The angle between $\boldsymbol{n}_{\boldsymbol{1}}$ and $\boldsymbol{n}_{\mathbf{2}}$ is denoted as $\alpha$. The maximum values of $|P Q|$ and $\alpha$ correspond to 'max distance' and 'max angle' settings in PolyWorks, which define the searching area for points matching.

The gap colormap changes with different values of max angle and max distance. Fig. 8 shows that if the max distance is set to $0.5 \mathrm{~mm}$, the colormap does not cover the whole surface. Fig. 9 shows if the max angle is set to 1 degree, the colormap is not continuous.

The parameter settings are briefly introduced as follows. Firstly, feeler gauges can be used to measure the gap, and obtain an estimation of maximum gap value. Then in PolyWorks, the value is set as max distance and adjusted until the colormap covers the whole surface and is continuous, whereas the max distance and max angle should be as small as possible, in order to define smaller but accurate search area. A number of experiments were conducted in PolyWorks in order to find out their appropriate values, i.e., max distance of $1.2 \mathrm{~mm}$, and max angle of 10 degree . Due to space limitation, the detailed decision making processes are not introduced here.

\section{2) Impact of sphere targets}

The standard deviations of gap measurement results in PolyWorks are calculated in order to find the impacts of using sphere targets, as shown in Table III. Results show that using sphere targets will not have much influence on the standard deviation of gap measurement results, but to improve registration efficiency, it is recommended to use 1 or 3 spheres as targets. However, it should be noted that the more targets used, the more points to be collected, which means measurement time and complexity will increase.

\section{3) Measurement results calibration and comparison}

In this test, twenty gap measurement positions were selected along the edges of the assembly, as illustrated in Fig. 10. Then the gaps between mating surfaces were manually measured with feeler gauges. In PolyWorks, the gaps were measured digitally, and were then calibrated by the systematic error of the laser scanner. Four groups of data were used in this test, i.e. scanned data with $0,1,2,3$ spheres. Comparative results are shown in Fig. 11 and Table III (mean error and mean error after calibration).

Fig. 11 shows that the digital and manual measurement results have the same trend, and the corrected values are closer to manual measurement results. This indicates that PolyWorks measurements are reliable once the measurement uncertainty was evaluated. Table III shows the mean errors between PolyWorks and feeler gauges, and the mean errors after calibration by Eqn. (6). Based on the results, it is always suggested to do accuracy calibration to remove systematic error in digital measurements.

\section{CONCLUSION}

The present study was designed to investigate registration uncertainty for precise aircraft wing assembly. This research is the first time to explore registration uncertainty for high precision assembly by using PolyWorks software. It gives the user insights on what should be considered when conducting

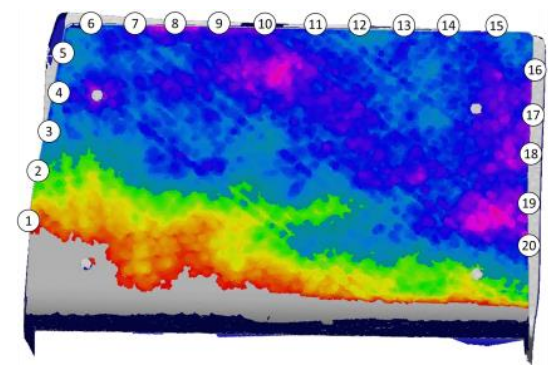

Fig. 10. Illustration of measured point positions
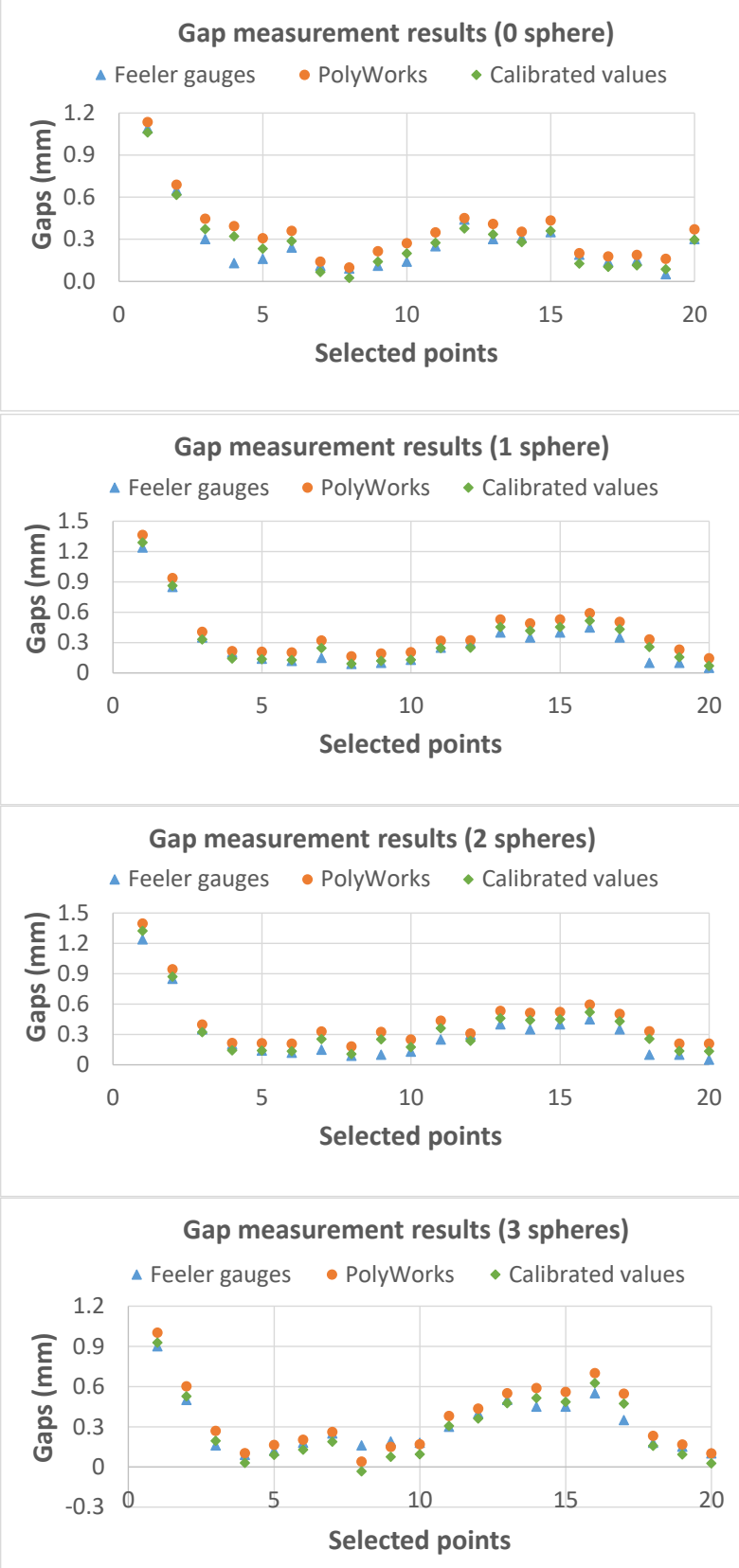

Fig. 11. Gap measurement results 
registration and gap measurements in PolyWorks. Conclusions are drawn as follows:

- Registration uncertainty has significant effects on gap measurement uncertainty.

- Using sphere artefacts as registration targets can help improve efficiency by reducing iteration times, but cannot improve registration uncertainty.

- Systematic errors should be carefully identified and applied to calibration, as they will affect the accuracy of the measurement not only at part-level but also at assembly-level, and their effects depend on the geometrical relationships of parts and assembly during registrations.

- Gap measurement results are carried both digitally in PolyWorks and manually by feeler gauges. The systematic error of the laser scanner is evaluated and eliminated in measurement results. Results show that the calibrated computational results are well aligned to manual measurement results.

- Max distance and max angle settings should be carefully set for obtaining accurate results in PolyWorks.

Further research will explore how to improve registration accuracy for high precision assembly considering components compliance. Metrology instruments with higher accuracy would be utilised.

\section{ACKNOWLEDGMENT}

The authors would like to acknowledge the Northern Ireland Technology Centre for using the equipment and software. Funding support from the Engineering and Physical Sciences Research Council (EPSRC) UK under projects EP/P025447/1 and EP/4P026087/1 is acknowledged. PhD funding support from Chinese Scholarship Council and Queen's University Belfast is also acknowledged.

\section{REFERENCES}

[1] H. Liu, X. Zhang, Y. Xu, and X. Chen, "Efficient Coarse Registration of Pairwise TLS Point Clouds Using Ortho Projected Feature Images," ISPRS Int. J. Geo Inf., vol. 9, p. 255, 2020.

[2] A. Eriksson, E. Sedelius, J. Berglund, and B. Johansson, "Virtual factory layouts from 3D laser scanning - A novel framework to define solid model requirements," Procedia CIRP, vol. 76, pp. 36-41, 2018/01/01/ 2018, doi: https://doi.org/10.1016/j.procir.2018.01.013.

[3] E. Minnetti et al., "Smart portable laser triangulation system for assessing gap and flush in car body assembly line," in 2019 II Workshop on Metrology for Industry 4.0 and IoT (MetroInd4. 0\&IoT), 2019: IEEE, pp. 49-53.

[4] A. Baleani, P. Castellini, P. Chiariotti, N. Paone, and L. Violini, "Analysis of reproducibility and repeatability of a handheld laser scanner for gap\&flush measurement in car-assembly line," in 2020 IEEE International Workshop on Metrology for Industry 4.0 \& IoT, 2020: IEEE, pp. 648-653.

[5] A. Gawel, R. Dubé, H. Surmann, J. Nieto, R. Siegwart, and C. Cadena, "3d registration of aerial and ground robots for disaster response: An evaluation of features, descriptors, and transformation estimation," in 2017 IEEE International Symposium on Safety, Security and Rescue Robotics (SSRR), 2017: IEEE, pp. 27-34.

[6] H. Sun, B. Luo, L. Yu, X. Ma, and L. Yan, "Mosaic research with 3D lidar point cloud of civil aircraft," in 2013 2nd International Symposium on Instrumentation and Measurement, Sensor Network and Automation (IMSNA), 2013: IEEE, pp. 683-686.
[7] H. Sun, J. Han, C. Wang, and Y. Jiao, "Aircraft model reconstruction with image point cloud data," in 2018 IEEE 3rd International Conference on Cloud Computing and Big Data Analysis (ICCCBDA), 2018: IEEE, pp. 322325.

[8]Y. Wang et al., "Density-invariant registration of multiple scans for aircraft measurement," IEEE Transactions on Instrumentation and Measurement, vol. 70, pp. 1-15, 2020.

[9] K. Pulli, "Multiview registration for large data sets," in Second International Conference on 3-D Digital Imaging and Modeling (Cat. No.PR00062), $8-8$ Oct. 1999 1999, pp. 160-168, doi: 10.1109/IM.1999.805346.

[10]Y. Liu, W. Zhou, Z. Yang, J. Deng, and L. Liu, "Globally consistent rigid registration," Graphical Models, vol. 76, no. 5, pp. 542-553, 2014/09/01/ 2014, doi: https://doi.org/10.1016/j.gmod.2014.04.003.

[11] Y. Tang and J. Feng, "Hierarchical multiview rigid registration," in Computer Graphics Forum, 2015, vol. 34, no. 5: Wiley Online Library, pp. 77-87.

[12] J. Xu, R. Chen, H. Chen, S. Zhang, and K. Chen, "Fast Registration Methodology for Fastener Assembly of Large-Scale Structure," ITIE, vol. 64, no. 1, pp. 717-726, 2017, doi: 10.1109/TIE.2016.2599140.

[13] L. Cheng et al., "Registration of Laser Scanning Point Clouds: A Review," (in eng), Sensors (Basel), vol. 18, no. 5, p. 1641, 2018, doi: $10.3390 / \mathrm{s} 18051641$.

[14] G. K. Tam et al., "Registration of 3D point clouds and meshes: A survey from rigid to nonrigid," IEEE transactions on visualisation and computer graphics, vol. 19, no. 7, pp. 1199-1217, 2012.

[15] P. J. Besl and N. D. McKay, "A method for registration of 3-D shapes," ITPAM, vol. 14, no. 2, pp. 239-256, 1992, doi: 10.1109/34.121791.

[16] J. Yang, Z. Cao, and Q. Zhang, "A fast and robust local descriptor for 3D point cloud registration," Information Sciences, vol. 346, pp. 163-179, 2016.

[17] J. Salvi, C. Matabosch, D. Fofi, and J. Forest, "A review of recent range image registration methods with accuracy evaluation," Image and Vision computing, vol. 25, no. 5, pp. 578-596, 2007.

[18] Y. Rajendra et al., "Evaluation of Partially Overlapping 3D Point Cloud's Registration by using ICP variant and CloudCompare," The International Archives of Photogrammetry, Remote Sensing and Spatial Information Sciences, vol. 40, no. 8, p. 891, 2014.

[19] B. Maiseli, Y. Gu, and H. Gao, "Recent developments and trends in point set registration methods," JVCIR, vol. 46, pp. 95-106, 2017.

[20] M. Bueno, H. González-Jorge, J. Martínez-Sánchez, L. Díaz-Vilariño, and P. Arias, "Evaluation of point cloud registration using Monte Carlo method," Measurement, vol. 92, pp. 264-270, 2016.

[21] M. Attia, Y. Slama, and M. A. Kamoun, "On performance evaluation of registration algorithms for 3d point clouds," in 2016 13th International Conference on Computer Graphics, Imaging and Visualization (CGiV), 2016: IEEE, pp. 45-50.

[22] P. Li, R. Wang, Y. Wang, and W. Tao, "Evaluation of the ICP Algorithm in 3D Point Cloud Registration," IEEE Access, vol. 8, pp. 68030-68048, 2020.

[23] T. Urbančič, Ž. Roškar, M. Kosmatin Fras, and D. Grigillo, "New Target for Accurate Terrestrial Laser Scanning and Unmanned Aerial Vehicle Point Cloud Registration," Sensors, vol. 19, no. 14, p. 3179, 2019. [Online]. Available: https://www.mdpi.com/1424-8220/19/14/3179.

[24] B. Becerik-Gerber, F. Jazizadeh, G. Kavulya, and G. Calis, "Assessment of target types and layouts in 3D laser scanning for registration accuracy," Automation in Construction, vol. 20, no. 5, pp. 649-658, 2011/08/01/ 2011, doi: https://doi.org/10.1016/j.autcon.2010.12.008.

[25] D. Yun, S. Kim, H. Heo, and K. H. Ko, "Automated registration of multi-view point clouds using sphere targets," Advanced Engineering Informatics, vol. 29, no. 4, pp. 930-939, 2015/10/01/ 2015, doi: https://doi.org/10.1016/j.aei.2015.09.008.

[26] Y. Wang, H. Shi, Y. Zhang, and D. Zhang, "Automatic registration of laser point cloud using precisely located sphere targets," Journal of applied remote sensing, vol. 8, no. 1, p. 083588, 2014.

[27] W. Liu, "Novel method for sphere target detection and center estimation from mobile terrestrial laser scanner data," Measurement, vol. 137, pp. 617$623,2019$. 